\title{
Assessment of Serum Level of Interleukin-17 in Patients with Vitiligo
}

N.W.Mikhael ${ }^{1}$, A.M. Hamed ${ }^{2}$, A.A. Elfallah ${ }^{3}$ and A.A. Ahmed $^{4}$

Dermatology, Venereology and Andrology Dept., Faculty of Medicine, Benha Univ., Benha, Egypt

E-Mail: aliaa_abdelsalam89@yahoo.com

\begin{abstract}
Vitiligo is a multifactorial disorder related to both genetic and non-genetic factors. The pathogenesis of vitiligo is associated with the melanocytes destruction that may result from immune and inflammatory mediators. The autoimmune theory is the most important theory about the pathogenesis of vitiligo. The aims of the study are the evaluation and assessment of serum levels of IL17 in vitiligo patients to reveal their possible role in pathogenesis of vitiligo and to correlate their levels with the severity of the disease. Patients and Methods: In this study, thirty patients (15 male and 15 females) were included in the present study and 30 apparently healthy, age and sex matched individuals were included as a control group. Patients were recruited from the outpatient clinic of Benha University Hospitals from Dermatology, Venereology and Andrology Department. All patients were subjected to full history, full dermatological and general examination. Measurement of serum levels of IL-17 using the commercially available ELISA kit. Results: The present study revealed significant increase in serum levels of IL-17 in patients than controls. Conclusion: Vitiligo is associated with increased serum level in IL-17 indicating that IL-17 may play a significant role in the pathogenesis of vitiligo.
\end{abstract}

Keywords: Vitiligo, Interleukin 17, enzyme-linked immunosorbent assay.

\section{Introduction}

Vitiligo is an idiopathic, acquired disorder that is characterized by circumscribed depigmented macules and patches which affect approximately $0.1-2 \%$ of the general global population. Vitiligo may appear any time from after birth to senescence. The average onset age of vitiligo is variable but its peak is almost in the second and third decades. The exact pathogenesis of vitiligo is still unclear and uncertain [1].

Vitiligo is a multifactorial disorder related to both genetic and non-genetic factors [2]. The pathogenesis of vitiligo is associated with the destruction of melanocytes resulting from immune and inflammatory mediators [3].

Interleukin-17 is a family of six cytokines that includes IL-17A through IL-17F [4]. It has been implicated in the pathogenesis of many immunemediated diseases [5].

The aim of this work is to evaluate and assess serum levels of IL-17 in vitiligo patients to reveal their possible role in pathogenesis of vitiligo and to correlate their levels with the severity of the disease.

\section{Material and methods}

The present study is a case-control study. It was conducted on thirty outpatient clinic of Dermatology, Venereology and Andrology Department of Benha University Hospitals suffering from Vitiligo (patients group) and thirty apparently healthy individuals of matched age and sex (Control group). This study was approved by the Local Ethics Committee on research involving human subjects of Benha Faculty of Medicine. An informed consent was obtained from each individual before being enrolled in the study and before sample collection.

\subsection{Inclusion Criteria:}

- Both genders were included.

- Patients those are willing and able to understand and sign informed consent.

\subsection{Exclusion Criteria:}

- Patients receiving systemic or topical anti-vitiligo therapy one month prior to the study.

- Patients with any conditions that could affect IL17 levels such as any acute inflammatory disease.

All included patients were subjected to full history taking including, personal history, present history of vitiligo; age of onset, course, duration of the disease and relation to stress or smoking, Past history of previous disease and previous history of treatment or medications and family history of vitiligo or other autoimmune diseases. Local examination for dignosis of vitiligo type and extent of the disease.

\subsection{Laboratory investigations:}

Five millimeters of venous blood samples were collected from each patient and control subjects by clean venipuncture using disposable plastic syringe. Samples were then dispensed in plain tube without anticoagulant for serum separation. The tubes were left to clot for twenty minutes at room temperature and then centrifuged at 3000rpm for ten minutes. The supernatant was carefully collected. The collected sera were finally stored at $-20^{\circ} \mathrm{C}$ to preserve until IL-17 testing.

Measuring serum level of IL-17 in the patients and Controls was carried out using double-antibody sandwich enzyme-linked immunosorbent assay (ELISA) method according to the manufacturer's instructions [6]. 


\subsection{Statistical Analysis}

Data management and statistical analysis were carried out using SPSS vs.25 (IBM, Armonk, New York, United states). Numerical data was summarized as standard deviations and means or ranges and medians. Comparisons between cases and controls were carried out by independent t-test for numerical data. While categorical data was compared using Chi-square or Fisher's exact test. Serum levels of IL-17 were compared within cases group as regard different study parameters using Independent t-test or Mann Whitney U-test and were compared as regard disease severity and activity using Kruskal Wallis test.

\section{Results}

Descriptive statistics of vitiligo patients and control group showed that there was no statistically significant difference regarding age, sex or smoking status between patients and control groups $(\mathbf{P}>\mathbf{0 . 0 5})$. Patients and controls were age-matched. The mean age \pm SD in vitiligo group was $25.9 \pm 3.43$ years, whereas mean age \pm
SD in control group was $27.80 \pm 5.65$ years. Clinical assessment of studied patients revealed that 20 patients $(66.6 \%)$ had positive family history of vitiligo while only one individual $(3.3 \%)$ of control group had positive family history. The results also revealed that eight patients with sudden onset of vitiligo. Fifteen patients $(50 \%)$ had progressive course of vitiligo. The duration of vitiligo disease in patients ranged from 8 months to 7 years.

There was statistically significant increase in serum levels of IL-17 in patients than controls with IL-17 mean \pm SD of $274.45 \pm 78.23(\mathrm{pg} / \mathrm{ml})$ for the vitiligo group and mean \pm SD of $117.57 \pm 65.18(\mathrm{pg} / \mathrm{ml})$ for the control group.

Non significant difference in serum levels of IL-17 regarding gender, smoking status.

Results of ROC curve showed that sensitivity, specificity and accuracy of serum levels of IL-17 for discrimination between patients and controls were $98.4 \%, 83.6 \%$ and $93.4 \%$ when the cutoff point was $>128.5$ (pg/ml) Table (1), Fig. (1).

\section{ROC Curve}

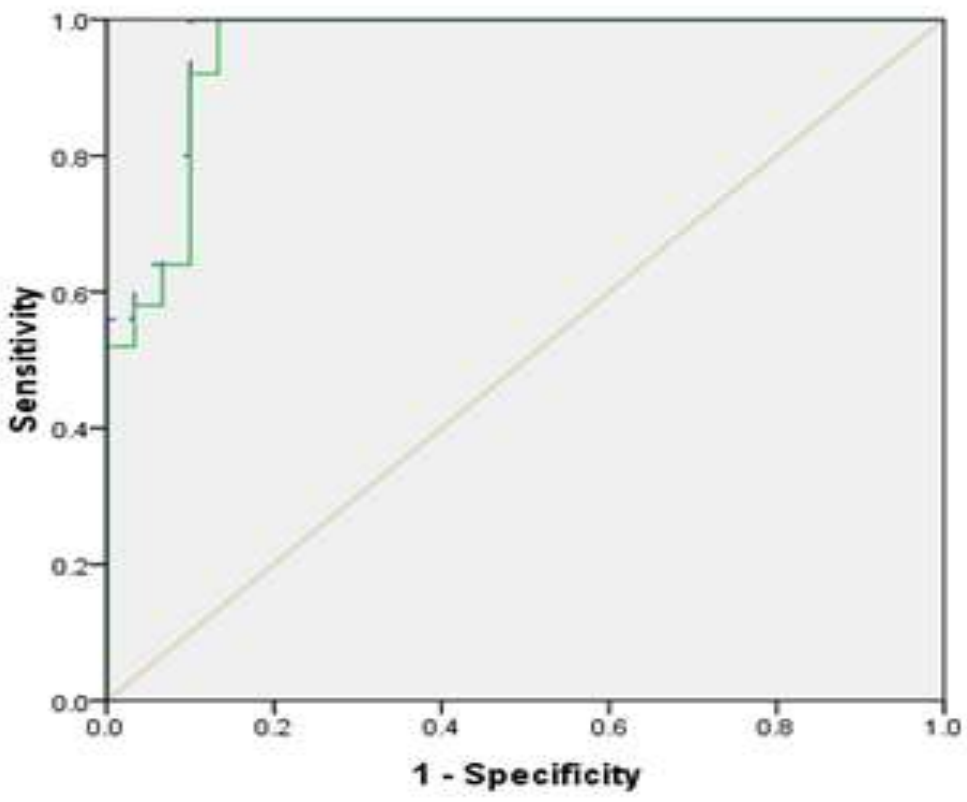

Source of the Curve

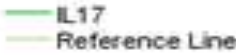

Fig. (1) ROC curve for the performance of serum IL-17 in early diagnosis (prediction) of vitiligo to discriminate between cases and control.

Table (1) Area under ROC curve and the performance features of serum IL-17 for discrimination between patients and controls.

\begin{tabular}{|l|c|l|l|l|l|l|l|}
\hline Variable & Cut off & AUC & Sens \% & Spec \% & PPV \% & NPV \% & Accuracy \\
\hline IL-17 & $>128.5(\mathrm{pg} / \mathrm{ml})$ & 0.85 & 98.4 & 83.6 & 92.59 & 100 & 93.4 \\
\hline
\end{tabular}




\section{Discussion}

Vitiligo is a multifactorial disorder related to both genetic and non-genetic factors [2]. The exact etiopathogenesis of vitiligo is still a controversial issue. Both cellular and humoral immune responses are thought to be responsible for a cascade of inflammatory reactions that mediate the destruction and loss of melanocytes. Overproduction of proinflammatory cytokines has been proved in vitiligo [3].

In the present study, there was statistically significant increase in serum IL-17 level in patients than controls $(\mathbf{p}<\mathbf{0 . 0 0 0 1})$ suggesting an important role of IL17 in pathogenesis of vitiligo. These results were in agreement with Habeb et al. (2013) [7].

The increased serum levels of IL-17 in vitiligo patients may be an indication of T-cell activation. In addition to IL-17, Th 17 cells secrete IL-6 and TNFa and also stimulate keratinocyte release of IL-1a, IL-6 and TNF-a. IL-17 itself also synergizes with these local inflammatory mediators, which may cause further inhibition of melanocyte proliferation [8].

On the other hand, Jandus et al. (2008) studied the role of Th17 cells in the pathogenesis of certain autoimmune disorders, including five patients with vitiligo, but did not find increased levels of Th17 cells in peripheral blood of patients with vitiligo compared with controls [9]. Basak et al. (2009) also reported insignificant difference between the patient and control groups in mean levels of IL-17 [10]. This contradiction of these results could be attributed to the different number of patients and control subjects enrolled which was not sufficiently powered. The geographic and cultural diversity could also account for such variation in results.

Sorour et al. (2020) enrolled a study on cases with stable nonsegmental vitiligo of more than one year versus cases with history of progression over the past three months [11]. They reported that serum levels of IL17 showed a non significant correlation with disease activity in the same direction of results of the study sparkled by Aly et al. (2017) [12].

Serarslan et al. (2020) detected no relation between serum levels of interleukin 17 in patients with extent of the vitiligo [13]. Aly et al. (2017) reported an insignificant negative correlation between IL-17 and extent of the disease on contrary to results of the present study [12]. These contradictory results may be due to different sample size.

There was non significant difference of serum levels of IL-17 between male and female patients, this going in line with the results of Bassiouny\& Shaker, (2011) [14] and Aly et al. (2017) who reported that serum IL-17 levels showed a non significant difference as regards gender [12].

\section{Conclusion}

Within the limitation of the present study, it was concluded that vitiligo is associated with increased serum level in IL-17 indicating that IL-17 may play a significant role in the pathogenesis of vitiligo.

Further studies are needed including larger number of patients for better evaluation of the relation between serum levels of IL-17 and Vitiligo. More studies are needed to evaluate serum levels of IL-17 of Vitiligo in patients before and after Vitiligo treatment. More investigations are needed to investigate the precise mechanisms by which serum levels of IL-17 contribute to the pathogenesis of vitiligo.

\section{References}

[1] M.Misterska, J.Szulczyńska-Gabor, R.Żaba, Aetiopathogenesis.clinical picture and treatment of vitiligo. Postep Dermatol. Alergol, vol.26(4).pp.212223, 2009.

[2] D.Kartal, M.Borlu, S.L.Çınar, et al., Thyroid abnormalities in pediatric patients with vitiligo: retrospective study. Postepy Dermatol. Alergol. vol.33(3).pp.232-234, 2016.

[3] M.Sandoval-Cruz, M. García-Carrasco, R. Sánchez-Porras et al., Immunopathogenesis of vitiligo.Autoimmun Rev.vol.10(12).pp.762-765, 2011.

[4] R.P. Singh, S. Hasan, S. Sharma et al., Th17 cells in inflammation and autoimmunity. Autoimmun Rev.vol.13.pp.1174-1181, 2014.

[5] R.K. Singh, K.M. Lee, I. Vujkovic-Cvijin et al., The role of IL-17 in vitiligo: A review. Autoimmun Rev.vol.15(4).pp.397-404, 2016.

[6] T.O.Kohl and C.A.Ascoli, Immunometric Antibody Sandwich Enzyme-Linked Immunosorbent Assay. Cold Spring HarbProtoc.vol.1;(6).pdb.Prot 093716, 2017.

[7] A.M. Habeb, A.M. Al Hefnawy, S.B. Elsayed et al., Expression of Interleukin-17 mRNA in Vitiligo Patients.Egy.J.Dermatol.Venerol.vol.33.pp.67-60, 2013.

[8] P.Miossec, IL-17 and Th17 cells in human inflammatory diseases. Microbes Infect.vol.11.pp.625-630, 2009.

[9] C. Jandus, G. Bioley, J.P. Rivals., Increased numbers of circulating polyfunctional Th17 memory cells in patients with seronegative spondyl arthritides. Arthritis Rhuem.vol.58.pp.2307-2317, 2008.

[10] P.Y. Basak, A.K. Adiloglu, A.M. Ceyhan et al., The role of helper and regulatory $\mathrm{T}$ cells in the pathogenesis of vitiligo. J.Am.Acad.Dermatol.vol.60 (2).pp.256-260, 2009.

[11] O.S. Sorour, E.S. Nassar, S.M. Eldin Ahmed et al., Serum levels of macrophage migration inhibitory factor and interleukin 17 in patients with 
nonsegmentalvitiligo. J.Egypt Womens Dermatol. Soc.vol.17.pp.13-18, 2020.

[12] D. Aly, F. Mohammed, K. Sayed et al., Is There a Relation between Vitamin D and Interleukin-17 in Vitiligo? A Cross-Sectional Study. Dermatology. Vol.233(6).pp.413-418, 2017.

[13] G. Serarslan, Z. Yönden, S. Söğüt et al., Macrophage migration inhibitory factor in patients with vitiligo and relationship between duration and clinical type of disease. Clin.Exp.Dermatol.vol. 35.pp.487-490, 2010.

[14] D.A. Bassiouny, O. Shaker, Role of interleukin-17 in the pathogenesis of vitiligo. Clin.Exp.Dermatol. vol.36 (3).pp.292-297, 2010. 